\title{
Design and Implementation of the Underground Pipeline Inspection System Based on Android
}

\author{
Xiaolin $\mathrm{Li}^{1}{ }^{1,}$, ${ }^{*}$, Guozhen $\mathrm{Shi}^{1}$, Siman $\mathrm{He}^{1}$, Meng Yang ${ }^{2}$, Changchun Zhou ${ }^{1}$ \\ ${ }^{1}$ Beijing Electronics Science and Technology Institute, Beijing, 100070, China \\ ${ }^{2}$ China Aerospace Science and Industry Corporation, the second Institute, Beijing, 100854, China \\ agxyy351@163.com
}

Keywords: Android, Inspection Terminal, GIS, Three-dimensional modeling, GPS.

\begin{abstract}
In recent years, urban underground pipeline accidents have occurred frequently, which caused huge losses of property and personal injury. In order to ensure the safe operation of urban underground pipeline and strengthen the urban underground pipe network in all directions, in this paper, using the Java language relying on Android Studio development platform, we designed and developed an underground pipeline inspection terminal based on Android platform, combined with GIS technology, three-dimensional modeling technology, GPS technology and 3G wireless communication technology. In addition, with a set of inspection management system deployed, the remote server is able to exchange information with the intelligent terminal. Analysis shows that the intelligent inspection system has the characteristics of visualizing the spatial level and location of the underground pipeline, accurate and rapid positioning accident, and controlling the underground pipeline accident risk, and is suitable for large-scale popularization and utilization.
\end{abstract}

\section{Introduction}

Urban underground pipelines are an indispensable part of urban infrastructure, which are like the "nerves" and "blood vessels" of our body. known as "the lifeline of the city" ${ }^{[1]}$. According to the statistical report of Underground pipeline committee of CACP(UPCC), around 5.6 underground pipeline explosion incidents occurred per day during 2008 to 2010. Among them, in November 2013, Qingdao oil pipeline explosion occurred, resulting in 62 people killed and 136 people injured, and direct economic losses of 751.72 million yuan. To this end, The State Council has made important instructions on the management of urban underground pipelines for several times. In June 2014, the State Council issued the Guidance on Strengthening the Management of Urban Underground Pipeline Construction. At the beginning of 2015, Premier Li Keqiang, Vice Premier Ma Kai and other national leaders made instructions clearly about strengthening the management of underground pipelines and build a "conscientious government".

According to statistics, the main cause of underground pipeline accidents is the external factors such as external damage, illegal operations, etc. Strengthen the regular inspection of underground pipelines can effectively reduce the frequency of accidents, and reduce the losses caused by the accident (especially in the early stages of the accident). However, Dozens of pipe such as water supply, drainage, gas, and so on, just arranged criss- cross, which increased the complexity of the distribution of underground pipelines, accordingly, the difficulty of the underground pipeline shift inspection was also increased greatly. Power transmission. Furthermore, the traditional way of shift inspection has many limitations, the position of the underground pipelines cannot be found accurately, .and it is difficult for managers to carry out supervision and assessment of shift inspection work, the only guarantee is the high sense of responsibility of inspectors ${ }^{[2]}$. Therefore, we need a new shift inspection system urgently to be used in the underground pipeline inspection.

Therefore, in this paper, we developed an underground pipeline intelligent inspection System based on the Android platform, which combined GPS (Global Positioning System) technology, GIS (Geographic Information System) technology and mobile communication technology. Using 3D modeling technology while combining map function and GIS function into mobile devices, this 
product can help the inspector find the location of the underground pipeline accurately, report the abnormal situation timely, and realize the real-time positioning of the inspector ${ }^{[3]}{ }^{[4]}$.

\section{OVERALL DESIGN OF SYSTEM}

\subsection{Overall Design of Framework}

In this paper, our underground pipeline inspection system used an architecture of combining M/S and B/S structure that is composed of the Mobile terminal (Mobile), the Browser (Browser), the Server (Server), the corresponding network and the communication link. Android mobile client side exchanges data with the background server via wireless communication, mainly be responsible for leading the inspector. The Service side is mainly responsible for information interaction with background database, manage the inspection tasks, events and other information, and provide mobile communication interface with the Web Service. The overall architecture of the system is shown in Fig.1.

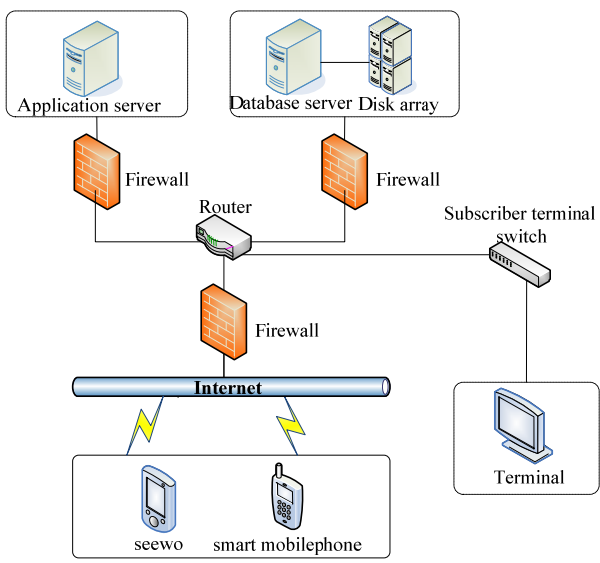

\subsection{Business Process Design}

Fig.1: Diagram of system overall architecture

The main business of the system is the daily inspection of underground pipelines and timely report of abnormal status, the entire system operation flow is shown in Fig.2. First, the inspection management staff assign tasks to the inspectors through the inspection management platform, then inspectors should login system through the mobile device and obtain work orders to carry out the task. In the course of performing tasks, inspectors should report abnormal events at the moment they are founded. After the inspection, inspectors should submit all work orders to complete the task.

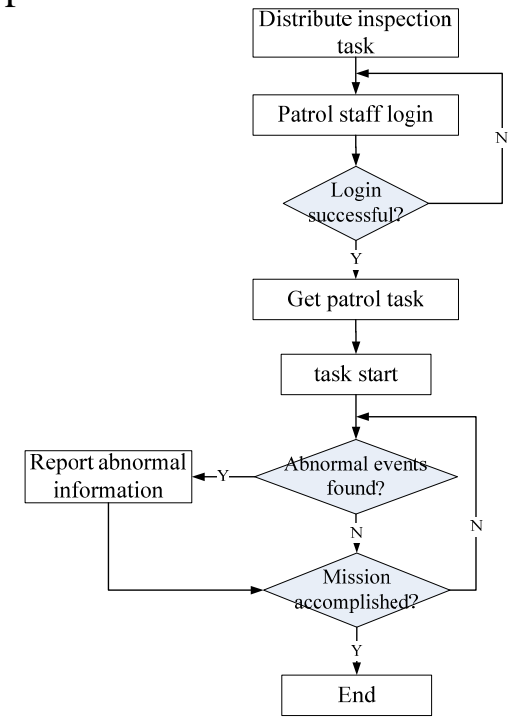

\subsection{Communication Method}

Fig.2: System Operation flow chart

At present, the common wireless communication methods are Bluetooth, ZigBee, Wi-Fi, mobile communication and so on. Among them, ZigBee has the characteristic of low power consumption 
and low cost, but its transmission distance is short. Wi-Fi has fast transmission speed, but its coverage is limited. As the current mainstream communications technology, $3 \mathrm{G}$ can carry out long-distance communication, and its downlink communication speed can be reached at 3.6Mbit/s, which is able to meet the general needs of communication. Taking the service environment of mobile terminal environment into account, we chose 3G communication eventually.

\section{FUNCTION DESIGN OF SYSTEM}

The underground pipeline inspection terminal based on Android includes two parts: server-side terminal and mobile terminal. The function modules of server-side terminal are mainly composed of inspection tasks management, event reporting management and inspection staffs management. It can achieve the following functions: assigning tasks to the mobile terminal, collecting the inspection route, checking the reported inspection events, and getting real-time positioning of the inspectors, etc. The function modules of mobile terminal mainly include inspection tasks management, three-dimensional pipeline browsing, inspection events reporting, inspection staff positioning, which can achieve functions like receiving inspection tasks, checking routing information and attribute information and other information of underground pipelines, taking pictures or videos to report inspection events, realizing the real-time positioning of inspectors , etc. The diagram of the system functions is shown in Figure 3. This article will focus on the design and implementation of Android mobile terminal.

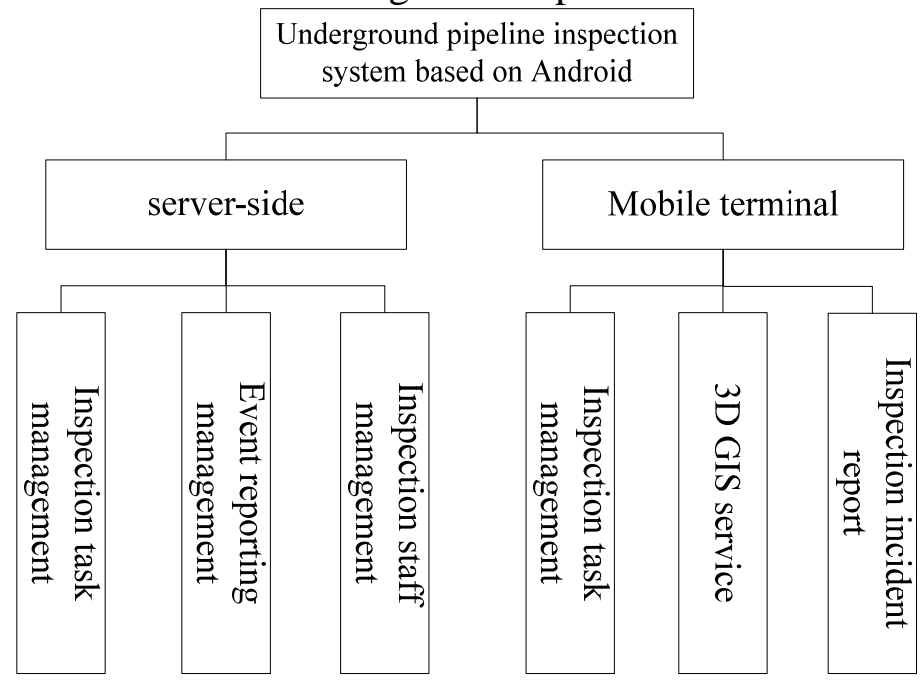

\subsection{D GIS Service Function}

Fig.3: Diagram of system functions

With the continuous development of GIS, resolving issues through three-dimensional space has gradually become a trend. Comparing to the two-dimensional GIS platform, the 3D GIS platform has great advantages in spatial location description and spatial analysis. The mobile terminal map function in the system is based on the API interface provided by the 3D GIS platform. The inspectors can carry out basic browsing, query and calculation on the map, and use the GPS module of the mobile terminal to view their current location, showing the tasks assigned by the administrator. Using 3D modeling technology, the system simulates current scene visually to facilitate inspectors making a better combination of virtual and reality in the process of the task. Among them, productions of the above ground buildings and pipeline model on the map are based on 3d Max (3D Studio Max). For the loading of the 3D model in Android terminal devices, the graphics rendering performance and memory requirements of the system must be high, luckily, the current mainstream Android devices on the market are supportable. The 3D GIS platform used in this article is provided by Beijing 3D Vision Technology Co., Ltd., which can quickly browse the 3D model and support 3D scene browsing of high volume data. Because of the less understanding of pipeline information during the underground pipeline inspection, therefore, in this system, we developed the function of adjusting the transparency of the map based on the API interface provided by the 3D GIS platform. By setting the ground to be transparent, inspectors can clearly see the distribution and routing of underground pipelines. In addition, by querying the pipeline information stored in the SQLite database that built- 
in Android, and combining with the GPS location information, the pipeline below the location of inspectors will be clear in their minds. Thus we can avoid inspectors from working blindly, and also improve the accuracy and efficiency of inspection greatly.

\subsection{Inspection Task Management Function}

As the underground pipeline inspection work is often done in the outdoor, we built the SIM card into the mobile device, so that inspectors can interact information with management platform through $3 G$ network. The data interaction between background inspection management system and mobile terminal equipment is realized base on SOA architecture, which is a kind of service-oriented architecture that can response to changing business requirements flexibly and rapidly. The background server opens communication interface for mobile terminal in Web Service way ${ }^{[5]}{ }^{[6]}$, it can authenticate users' identity and ensure the security of data through the way of the user login.

The manager assign tasks to inspectors through the inspection management platform, requiring inspectors to conduct inspection follow the provided route. After the inspector login the system and request for inspection tasks to the server, the background server will verify the identity of inspector and send task information to the client in the form of JSON. The data is parsed on the mobile terminal and send to inspectors.

The inspector should hold mobile device that can record their location in real time by GPS, once the system found that the inspector is deviate from the inspection route, it will send out alarm to prompt. During the inspection process, the mobile terminal equipment will report the inspector's location, time and other information to the inspection management platform through 3G network, so that the real-time monitoring of inspection can be achieve. In addition, by connecting the location points of the task, the trajectory of the mission can be clearly reflected in the map, which is the basis of assessment on inspectors' personnel work ${ }^{[7]}$.

\subsection{Inspection Incident Report Function}

In the process of underground pipeline inspection, the inspectors may find some unusual events such as gas leakage, water leakage, loss of manhole covers, illegal construction and other phenomena, then inspectors can report such events ${ }^{[8]}$. Through the handheld terminal device, inspectors can describe the accident information, and use its built-in camera to take photos or video of the accident, then report them and event location information obtained by GPS to inspection management platform through the $3 G$ network. According to the information reported by inspectors, managers can understand the situation of the accident at the first time, so that they can arrange the maintenance personnel to deal with the incident. The mobile terminal interface design is shown in Figure 4.

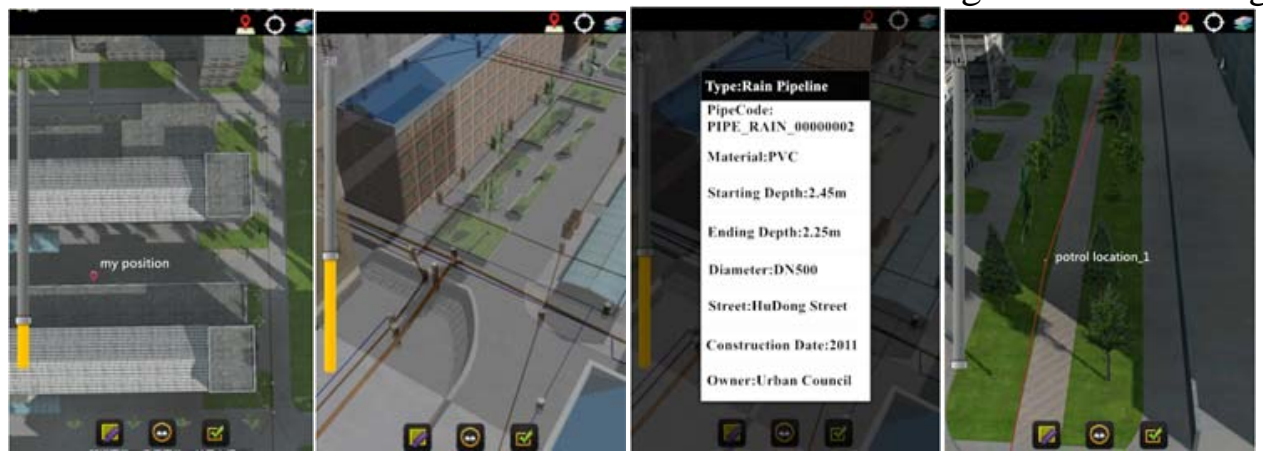

Fig.4: Interface design of mobile terminal

\section{Feedback of Practical Application}

The inspection system we designed has been put into practical application in many intelligent pipe network projects. In the course of more than one year of use, it has been constantly improved according to the user's feedback. At present, the system is in stable, strong operation, and achieved great results. The hardware and software of our system were applied outdoor, and tested in a variety of circumstances, it turns out that the positioning is accurate, the communication is normal, and displayed a good performance, achieved the height that the traditional way cannot reach out. 


\section{Summary}

For the frequent underground pipeline accidents, more and more attention has been paid to underground pipelines, and intelligent pipe network also came into being. In this paper, based on the Android system, combined with GPS, GIS and wireless communication technology, we developed an underground pipeline inspection system. With the application of three-dimensional modeling technology, the problems of low efficiency and difficulty in the traditional inspection mode has been solved. At the same time, it has become more convenient to manage the staff, which greatly improved the work efficiency and achieve a modern, intelligent work environment. More importantly, the application of our system will greatly reduce the frequency of underground pipeline accidents, and better to ensure the safe and stable operation of urban infrastructure. In short, our system has great application prospects in the field of intelligent pipe network.

\section{Acknowledgements}

This work was supported by the Beijing Natural Science Foundation under Grant No.4152048 and National Key Research and Development Project-the key technology and system of the world integrated network cipher service under Grant No.2016YFB0800304.

\section{References}

[1] Wu Yangan. Based on ArcGis for Android urban pipeline inspection system design and implementation of [J]. Bulletin of surveying and mapping, 2013:212-215.

[2] Chen Chuanjie, zhong-jun du. Embedded mobile patrol system based on PDA and GIS research [J]. Journal of measurement and control automation, 2009 (25): 159-160.

[3] z. Xu, X., w. Chen, G, X., Liu, et al: o Integrating mobile GIS, GPS, real - time D - and high resolution satellite imagery for land use patrolling [A]. In Proceedings Changes [C]. Of 2nd International Conference on Earth Observation for Szechwan Global Chengdu, China to Bellingham, WA, USA: SPIE, May 2009 v01. 7471, PP. 747123-1-747123-6.

[4] x. l. Liu, x. h. j. Tong, Ma. The Pipe patrol inspection system -based on Googleearth and $3 \mathrm{~g}$ integration technology [A]. In: Proceedings of 2008 International Workshop on Education technology and Training and 2008 International Workshop on A team and Remote Sensing [C]. Shanghai, China, Piscataway, USA: IEEE Computer Society, Dec. 2008, v01. 2, PP. 480-483.

[5] Tan Guomao gui-ming he. Checking system model and the technical scheme of the [J]. Journal of system construction, 2003 (1): 17-20.

[6] victory. Communications equipment monitoring system based on GPRS design [J]. Xi 'an: xi 'an university of electronic science and technology, 2005.

[7] harvest, zheng Li Jinjiang, tao xiang, Sun Bai. Gas intelligent inspection system design study [J]. Journal of surveying and mapping, Beijing, 2015 (5): 72-75.

[8] Zhao Jingsheng. City gas pipeline network intelligent inspection system of application study [J]. Journal of gas and heat, 2008, 28 (1): 801-805. 